\title{
Laser Therapy for Spinal Diseases
}

\author{
Jun Young Kim, II Sup Kim ${ }^{\bowtie}$, Jae Taek Hong, Jae Yeol Kwon, Jae Hoon Sung, Sang Won Lee \\ Department of Neurosurgery, The Catholic University of Korea, St. Vincent's Hospital, Suwon, Korea
}

\begin{abstract}
Since its introduction in 1980s, laser therapy, a minimally invasive, outpatient-based, effective, and relatively safe procedure for spine disease, has established itself world-wide. Percutaneous laser disc decompression (PLDD) was mainly applied to treat lumbar disc herniation with a satisfactory efficacy. Recently, the laser therapy has been used for various diseases including lumbar spinal stenosis and cervical disc diseases. In this article, the advantages and disadvantages of laser therapy will be discussed. Technical and clinical aspects of current applications for spinal diseases will be also presented. Such applications include percutaneous lumbar disk decompression (PLDD), percutaneous endoscopic laser annuloplasty (PELA), epiduroscopic laser neural decompression (ELND), and laser disc decompression in cervical disc diseases.
\end{abstract}

Key Words: Laser therapy; Percutaneous lumbar disc decompression; Percutaneous endoscopic laser annuloplasty; Epiduroscopic laser neural decompression.

\Corresponding author: II Sup Kim, Department of Neurosurgery, Catholic University of Korea, St. Vincent's Hospital, 93 Jungbu-daero, Paldal-gu, Suwon 16247, Korea. Tel: 82-31-249-7196, Fax: 82-31-249-5208, E-mail: nsman72@hanmail.net

\section{INTRODUCTION}

Laser therapy has been introduced for the treatment of spinal diseases since the 1980s. In February 1986, Choy et al. ${ }^{6}$ implemented percutaneous laser disc decompression (PLDD) on a lumbar herniated disc. Since then, the development of laser therapy for spinal diseases has focused on lumbar disc disease, and PLDD became an active treatment following FDA approval in $1991^{5)}$. Also, its application range has been expanding in recent times to include treatment of lumbar spinal stenosis and endoscopic treatment of ruptured cervical disc ${ }^{8,9)}$. This report aims to introduce the different methods of laser treatment and their effects on surgical treatment of spinal diseases.

\section{TYPES OF LASER THERAPY AND THEIR ADVANTAGES}

Laser therapy in spinal diseases is mostly carried out using the percutaneous and endoscopic approach. In this method, skin incision is minimized, as well as damage to the muscles, blood vessels, and nerves around the spine. It is also advantageous since operations are done under local anesthesia and the satisfaction of patients is high owing to the short operation time.

PLDD which is the oldest treatment method reduces discogenic pain by vaporizing the nucleus pulposus using an optical fi- ber. Percutaneous endoscopic laser annuloplasty (PELA) and epiduroscopic laser neural decompression (ELND) are also other types of laser therapy for spinal diseases. PELA is a method that conducts laser cauterization with an endoscopic approach to the annulus fibrosus of damaged intervertebral discs and ELND removes protruded discs and fiberized wounds using lasers with an extradural endoscope ${ }^{11,12)}$. In recent times, laser therapy using Cervical Deuk Laser Disc Repair ${ }^{\circledR}$ has been introduced for patients with ruptured cervical discs ${ }^{9}$.

The applicable laser types are Nd : YAG (neodymium-doped yttrium-aluminum-garnet laser) and Ho : YAG (holmium : yttrium-aluminum-garnet laser) in infrared light and the doublefrequency Nd: YAG laser and the potassium-titanyl-phosphate (KTP) laser in areas of green visible light. Of the above, the Ho : YAG laser and the ND : YAG laser are the most widely used.

\section{Percutaneous laser disc decompression (PLDD)}

The anatomic structure of disc is a closed hydraulic system, which serve as a basis for spinal disease treatment. Discs are composed of a nucleus pulposus with a high moisture composition and a surrounding annulus fibrosus. When the moisture proportion of the nucleus pulposus increases, the inner pressure on the discs increases. Choy et al. ${ }^{7)}$ reported that if the internal capacity of discs increases by $1.0 \mathrm{~mL}$, the pressure increases by about $312 \mathrm{kPa}$ (2340 mm Hg). On the other hand, if the internal ca- 
pacity of discs decreases, the pressure decreases as well. Radiating pain is caused by the pressurization of nerve roots due to lumbar disc herniation. Therefore, if the inner pressure on the discs is lessened, the herniated disc moves into the center, the pressurization of the nerve roots decreases, and the pain is alleviated. The main mechanism is to decrease pressure in the discs by vaporizing the moisture in the nucleus pulposus using lasers. Additionally, it has been reported that heightening the temperature in the nucleus pulposus decreases the receptive capacity of the moisture and the pressure inside the discs through the denaturation and regeneration of protein.

\section{Eligible patients}

1. Patients complaining of lumbago and radiating pain corresponding to radiologic tests.

2. Patients who experience no improvement of symptoms regardless of conservative treatment for a month or more.

3. Patients who have a medical disorder that would contraindicate general anesthesia, particularly elderly patients.

4. Patients with no bleeding tendency and metastatic lesions on the spine.

5. Patients with no severe scoliosis and retrolisthesis.

6. Patients with no mental illness, medication addiction, and/ or severe neuropathy.

\section{Operation method}

1. The operation is carried out under local anesthesia.

2. The correct disc level is checked using a $\mathrm{C}$-arm.

3. A needle is inserted in the skin about $10 \mathrm{~cm}$ apart from the midline toward the center of the disc.

4. The insertion of the needle into the center of the disc is checked in both directions using a C-arm fluoroscope.

5. Using the needle, the laser fiber is positioned in the center of the nucleus pulposus.

6. The pressure in the nucleus pulposus is decreased through the transmission of laser energy to the nucleus pulposus and the vaporization of its contents.

\section{Treatment results and complications}

Spinal surgery treatments are reported to have a success rate of between $70 \%$ and $90 \%$. However, the results of successful treatment are analyzed differently due to the variety of standard measurement. In most cases with minor improvements, free fragments were found in spinal canal. In about $4 \%$ to $20 \%$ of cases, it was reported that improvements in symptoms were slight or that herniation of a disc recurred ${ }^{1,4}$. While the herniation was minor in most cases, there were a number of cases where free fragments were found in the spinal canal.

The most common complication of PLDD is discitis, either of the septic or aseptic type. Aseptic discitis occurs as a result of thermal damage to the vertebral endplates. To prevent this complication, proper control in terms of power, frequency, and pulse gap is necessary. Since the purpose of PLDD is to vaporize the moisture in the nucleus pulposus, damage to the surrounding annulus fibrosus must be avoided. Thus, heat transmission using lasers should be at the lowest ranges and there should be a sufficient interval between heat transmissions as well. Also, there should be a sufficient gap between each bout of heat transmission. Septic discitis occurs due to intrusion of microbes when the needle is inserted. To prevent this, the treatment area should be disinfected as appropriate. There have also been reports that the additional antibiotics may helpful in the prevention of septic discitis .

\section{Limitations of PLDD}

Since PLDD decompresses the nucleus pulposus indirectly by using $\mathrm{C}$-arm fluoroscopy, this therapy has been found to be less effective than palliative surgery. Therefore, direct endoscopy using lasers and micro clamps can be used as an alternative method for removing lesions. Although PLDD has been investigated in many studies after used for the treatment of discogenic back pain, no randomized controlled test has been conducted. Additional studies using randomized control tests are needed to compare PLDD with traditional operation methods.

\section{Use of PLDD in spinal stenosis}

Ren et al. ${ }^{11)}$ reported that PLDD has a positive effect with regard to the treatment of spinal stenosis. The same mechanism mentioned above, lowering pressure in the nucleus pulposus by vaporizing using lasers, is applied. However, the treatment is less effective when the severe pressure presents on the back of spine by yellow ligaments in case of spinal stenosis. The treatment is effective only when the frontward pressure is dominant due to the discs.

\section{Percutaneous endoscopic laser annuloplasty (PELA)}

Even though the general process of discogenic pain is controversial, it is known that the pain results from internal disc disruption, degenerative disc changes, herniation of the nucleus pulposus, and damage to the annulus fibrosus ${ }^{10)}$. Peng et al. ${ }^{14)}$ reported that granulation tissue from the annulus fibrosus grows on the inside of disc and generates nerve damage, thus causing pain. The granulation tissue of the annulus fibrosus causes lumbago rather than radiation pain. Thus, Lee et al. ${ }^{12)}$ introduced the percutaneous endoscopic laser annuloplasty procedure, which cauterizes the granulation tissue related to the damaged annulus fibrosus. The main advantage of the PELA procedure is to preserve a healthy nucleus pulposus by minimizing damage to the center and front of the disc.

\section{Eligible patients}

1. Patients with lumbago for over two years who show no improvement despite conservative treatment for more than six months.

2. Patients who have undergone an MRI that showed disc degenerative changes. 
3. Patients complaining of discogenic lumbago where damage to the annulus fibrosus has been diagnosed in discography.

4. Patients who have a lesion in a segment.

5. In cases where the following conditions have been excluded : herniation, fragments, narrowness of a disc, instability of a segment, and multi-segment disc degeneration.

6. In cases where fracture, tumor, and infectious lesions have been excluded.

\section{Operation method}

1. The operation is carried out under local anesthesia.

2. The correct disc level is checked using a C-arm fluoroscopy.

3. Discography is implemented to check whether or not the annulus fibrosus is damaged and there are signs of lumbago.

4. If there is no damage to the annulus fibrosus and no inducible pain, surgery is stopped.

5. The entry point is made where a vertical line from the upper and lower facet joints and a horizontal line from the disc space meet. Normally, the entry point is usually outside area where is $12-15 \mathrm{~cm}$ apart from the midline.

6. When the needle enters in the direction of the facet joints, the axial angle is presented below 22 degrees.

7. Before the needle enters the annulus fibrosus, an endoscope should be used to check the extradural space, thecal sac, and nerve roots.

8. When the needle has entered the annulus fibrosus, damage to the annulus fibrosus should be checked using a contrast medium.

9. A catheter is inserted into the subligamentous inter-annular region of the annulus fibrosus.

10. The damaged annulus fibrosus is treated with cautery using Ho : YAG lasers.

11. Disc fragments should be removed using endoscopic forceps.

\section{Treatment results and complications}

Lee et al. ${ }^{12)}$ reported significant improvement of lumbago and statistically significant improvements in line with Macnab's criteria following PELA treatment. However, according to Carragee et $\mathrm{al}^{3)}$, only around $30 \%$ showed improvement. The frequency of specific complications or infection was not high. It is likely that damage to other parts was minimized by the short treatment time and use of the endoscope.

\section{Limitations of PELA}

Compared with PLDD, very little research has been carried out on this method, and randomized control tests have not been implemented. Also, evaluating pain through discography may be difficult to select a patient group appropriate for treatment since it can act as a psychogenic bias. Thus, in order to diagnose lumbago, it is necessary to check in detail for lesions, implement physical examinations, and obtain clear radiologic evidence through tests.

\section{Epiduroscopic laser neural decompression (ELND)}

In the case of patients complaining of lumbar and radiating pain that has not responded to conservative therapy, epiduroscopic laser neural decompression?is conducted using an extradural endoscope. For most patients, a nerve root block or palliative surgery is conducted. However, if these measures fail and there is no improvement after surgery, ELND is considered. It allows communication between a doctor and a patient under local anesthesia and exfoliation of adhesion in the epidural area. ELND also removes damaged tissue and inflammation and a protruded disc to lessen pressure on the nerves. Additionally, steroids and a topical anesthetic can be injected directly around a lesion. Hence, ELND can be a good therapy method for patients with various lesions.

Jo et al. ${ }^{11)}$ implemented ELND using Ho : YAG lasers. In their procedure, a $2100 \mathrm{~nm}$ wavelength was selected at the tissue to be removed. The laser was then inserted at a depth of $0.3-0.5 \mathrm{~mm}$ and the tissue was cauterized, thus minimizing damage to the surrounding tissue. However, since there are cases where the nerve roots are damaged or discitis occurs, caution is required for this procedure.

\section{Use of lasers in cervical disc disease}

\section{Use of PLDD}

Li et al. ${ }^{13)}$ have cured patients with cervical disc disease using PLDD. Targeting a total of 47 patients, the method was used to vaporize the nucleus pulposus of the cervical disc by approaching the front of the collum percutaneously. In around $75 \%$ of the patients, there was an improvement of symptoms, and overall, there were no complications. Thus, PLDD was introduced as a safe and non-invasive therapy method.

\section{Cervical Deuk Laser Disc Repair ${ }^{\circledR}$}

In 2012, Deukmedjian et al. developed a new anterior approach for treating cervical disc disease using an endoscope ${ }^{9)}$. The treatment is based on Ho : YAG lasers and is known to have an effect on degenerated disc disease, cervical spine, spinal stenosis, and damage to the annulus fibrosus. By approaching the cervical spine from the front and using an endoscope, posterior ligaments, posterior spinal endplates, annulus fibrosus, neuropores, and fragments of degenerated discs can be checked directly.

Cervical Deuk Laser Disc Repair ${ }^{\circledR}$ treatment can treat the disease while preserving discs. On the other hand, anterior cervical discectomy with fusion (ACDF) poses a risk in terms of worsening the stability of segments and causing kyphosis. In addition, ACDF can be dangerous because it may cause pressure on the neck and result in damage to the recurrent laryngeal nerves caused by instability of instruments, pseudoarthrosis, dysphagia, disability, contagion, and hematoma. However, Deukmedjian et al. reported no side effects of treatment using Cervical Deuk Laser Disc Repair ${ }^{\circledR}$ treatment. Its treatment indication is similar as the one of ACDF and targets the patients who complain of 
cervical pain, upper radiating pain, and general weakness and shows radiological defect related to the symptoms.

\section{Operation method}

1. The operation is carried out under general anesthesia.

2. The location of a lesion is checked using $\mathrm{C}$-arm fluoroscopy.

3. The needle is inserted opposite the area in which the symptom is present. The operation is carried out on the outside of the carotid and inside the esophagus and the respiratory tract.

4. C-arm fluoroscopy is used to position the tip of the needle at the front of the disc between the longus colli muscles and to place it into the center of the disc.

5. A dyeing material is used to facilitate endoscopic clarity throughout the procedure.

6. A guidewire is used to guide the endoscope to the inside of the disc. The posterior longitudinal ligament, posterior spinal terminalis, annulus fibrosus, neuropores, and fragments of the ruptured disc are then checked.

7. The damaged disc is recovered using Ho : YAG lasers and the Wolf (Germany) endoscopic forceps.

8. Bone spurs pressing against the spinal cord or neuropores can be cauterized for a few seconds, with the lasers set to $20 \mathrm{~W}$.

9. During the endoscopic treatment, continual cleaning with low-temperature physiological saline is necessary.

\section{CONCLUSION}

This research investigated the specific fields and methods of laser treatment generally used to address spine diseases. Overall, laser treatments have advantages over palliative operation treatment in terms of less invasive procedures, shorter treatment periods, and faster recovery times. Also, in a number of studies, while improvement of symptoms after the treatment appeared to be significant, the complication issues were minor. Therefore, it can be concluded that further research on laser treatments will be helpful in the treatment of spine diseases. More- over, because randomized control experiments have not been carried out in most studies, this is likely to be a necessity for future studies.

\section{REFERENCES}

1. Ascher PW : Laser trends in minimally invasive treatment : atherosclerosis, disk herniations. J Clin Laser Med Surg 9 : 49-57, 1991

2. Bosacco SJ, Bosacco DN, Berman AT, Cordover A, Levenberg RJ, Stellabotte $J$ : Functional results of percutaneous laser discectomy. Am J Orthoped 25 : 825-828, 1996

3. Carragee EJ, Lincoln T, Parmar VS, Alamin T : A gold standard evaluation of the "discogenic pain" diagnosis as determined by provocative discography. Spine (Phila Pa 1976) 31 : 2115-2123, 2006

4. Casper GD, Hartman VL, Mullins LL : Results of a clinical trial of the holmium : YAG laser in disc decompression utilizing a side-firing fiber : a two-year follow-up. Lasers Surg Med 19: 90-96, 1996

5. Choy DS : The true story of percutaneous laser disc decompression. J Clin Laser Med Surg $19:$ 231-233, 2001

6. Choy DS, Case RB, Fielding W, Hughes J, Liebler W, Ascher P : Percutaneous laser nucleolysis of lumbar disks. N Engl J Med 317 : 771-772, 1987

7. Choy DS, Michelsen J, Getrajdman G, Diwan S : Percutaneous laser disc decompression : an update--Spring 1992. J Clin Laser Med Surg 10 : 177 184,1992

8. Choy DS, Ngeow J : Percutaneous laser disc decompression in spinal stenosis. J Clin Laser Med Surg 16 : 123-125, 1998

9. Deukmedjian AJ, Cianciabella A, Cutright J, Deukmedjian A : Cervical Deuk laser disc repair : a novel, full-endoscopic surgical technique for the treatment of symptomatic cervical disc disease. Surg Neurol Int $3: 142$, 2012

10. Groen GJ, Baljet B, Drukker J : Nerves and nerve plexuses of the human vertebral column. Am J Anat 188 : 282-296, 1990

11. Jo DH, Yang HJ : The survey of the patient received the epiduroscopic laser neural decompression. Korean J Pain 26 : 27-31, 2013

12. Lee SH, Kang HS : Percutaneous endoscopic laser annuloplasty for discogenic low back pain. World Neurosurg 73 : 198-206, 2010

13. Li K, Qin H, Chen J : Clinical application of percutaneous laser disc decompression in the treatment of cervical disc herniation. Zhongguo Xiu Fu Chong Jian Wai Ke Za Zhi 21 : 465-467, 2007

14. Peng B, Wu W, Hou S, Li, P, Zhang C, Yang Y : The pathogenesis of discogenic low back pain. J Bone Joint Surg Br 87 : 62-67, 2005

15. Ren L, Han Z, Zhang J, Zhang T, Yin J, Liang X, et al. : Efficacy of percutaneous laser disc decompression on lumbar spinal stenosis. Lasers Med Sci 29 : 921-923, 2014 